\title{
Estresse em cuidadores de pacientes com Alzheimer: uma revisão de literatura
}

\author{
Stress in caregivers of Alzheimer's disease: a literature \\ review
}

Kamilla Sthefany Andrade de Oliveira*

Universidade Federal do Rio Grande do Norte - UFRN, Natal, Rio Grande do norte, Brasil

\section{Marianna Carla Maia Dantas de Lucena**}

Universidade Federal do Rio Grande do Norte - UFRN, Natal, Rio Grande do norte, Brasil

\section{J oão Carlos Alchieri***}

Universidade Federal do Rio Grande do Norte - UFRN, Natal, Rio Grande do norte, Brasil

\begin{abstract}
RESUMO
Verifica publicações referentes à temática do estresse em cuidadores de pacientes com Alzheimer, a partir de uma análise das publicações dos anos de 2006 a abril de 2013, sendo os mais recentes em termos de publicação na área. Método: revisa sistematicamente através de buscas nas bases de dados: Scielo, Bireme, PubMed com descritor: "estresse em cuidadores de Alzheimer", escrito em inglês e Português. Resultados: encontra 32 artigos que estavam dentro dos critérios de inclusão, sendo avaliados com base em uma análise temática acerca de questões práticas da atividade, aspectos físico-patológicos e alternativas de intervenções. Dos 32 artigos, 9 se referiam a estudos da prática do cuidar; 13 tratavam dos aspectos físicospsicopatológicos; e 9 sobre intervenção. Conclusão: conclui que o estresse nos cuidadores é um ponto que se expande em muitos outros problemas que desencadeiam comprometimentos na qualidade de vida, do ponto de vista físico, mental, psicológico e emocional.
\end{abstract}

Palavras-chaves: estresse fisiológico, cuidadores, doença de Alzheimer.

\section{ABSTRACT}

Check publications related to the topic of stress in caregivers of patients with Alzheimer's, from an analysis of publications from 2006 to April 2013, being the latest in terms of publication in the area. Method: A systematic review by searching the following databases Scielo, Bireme, PubMed with the descriptor: "Stress in caregivers of Alzheimer's;" written in English and Portuguese. Results: 32 articles were found and were within the inclusion criteria and were assessed based on a thematic analysis on issues of practical activity, physical and pathological aspects of alternative interventions. 9 of the 32 articles referred to the practice of care, 13 dealt about the physical-psychopathological, and 9 on interventions. Conclusion: 
the stress on caregivers is a point that expands into many other problems that trigger impairments in quality of life, from the standpoint of physical, mental, psychological and emotional.

Keywords: physiological stress, caregivers, Alzheimer disease.

\section{Introdução}

A população do Brasil, e do mundo como um todo, está ficando mais velha. Segundo dados do censo demográfico brasileiro do Instituto Brasileiro de Geografia e Estatística (IBGE) as projeções para o ano de 2020 são de que os idosos atingirão 25 milhões de indivíduos, destes 15 milhões serão mulheres, e representarão $11,4 \%$ da população total (IBGE, 2003). Com o envelhecimento da população há uma maior incidência de doenças crônicas degenerativas, entre elas as demências, que atingem de 3 a $11 \%$ das pessoas com mais de 65 anos e de 20 a $50 \%$ dos idosos com mais de 85 anos, sendo a mais frequente a Doença de Alzheimer (DA) (Machado, 2006). A DA constitui-se em uma patologia neurológica degenerativa progressiva e irreversível, que tem início insidioso e é marcada por perdas graduais da função cognitiva e distúrbios do comportamento e afeto. A doença começa com manifestações lentas com evolução progressiva e deteriorante. (Townsend, 2009 apud Almeida, 2009; Leite \& Hildebrandt, 2009).

Envolve o sistema nervoso central (SNC), de modo que afeta negativamente os processos de memória, mente e comportamento, com consequente perda da capacidade de executar atividades normais do cotidiano e, portanto, perda da autossuficiência (Ferrara Langiano, Brango, Vito, Cioccio \& Bauco, 2008). Normalmente o primeiro aspecto clínico é a deficiência da memória recente, com preservação das memórias remotas, somando-se às dificuldades de atenção e fluência verbal. Em sua progressão surgem dificuldades como a diminuição da capacidade de fazer cálculos, habilidades vísuo-espaciais e da capacidade de utilizar objetos comuns e ferramentas (Lindeboom \& Weinstein, 2004), exigindo cada vez mais cuidados (Cruz \& Hamdan, 2008). A deterioração das habilidades cognitivas e físicas, muitas vezes associada a sintomas cognitivos, tais como sintomas psicóticos, depressão e mudanças de comportamento, típicos desta doença, resultam em um pesado fardo para os que lidam diariamente com esses pacientes, representando um problema de saúde, econômico e social (Ferrara et al.,2008).

A definição de cuidador é muito discutida, este já foi considerado como o membro da família que provia cuidados ao paciente, outrora definido como o indivíduo que residia com o paciente e que atuasse no cuidado por mais de seis meses, ou ainda, como o principal responsável por prover ou coordenar os recursos requeridos pelo 
paciente (Cassis, Karnakis, Morais, Curiati, Quadrante \& Magaldi, 2007). Essa concepção continua a ser um assunto de intenso debate, não havendo um consenso (Cruz \& Hamdan, 2008). Para tanto, "o cuidador é um indivíduo que assume a responsabilidade de cuidar, dar suporte ou assistir alguma necessidade do enfermo, visando a melhoria de sua saúde" (Leitão \& Almeida, 2000, p. 80). Pode ser ainda um indivíduo que presta atendimento de forma temporária ou permanente, com função de auxílio nas incapacidades funcionais (Nascimento, Moraes, Silva, Veloso \& Vale, 2008).

As mudanças nos níveis de habilidades e comportamentos podem requerer excessiva atenção, expondo assim o cuidador ao risco de depressão e vulnerabilidade física, especialmente quando não há apoio de outros membros familiares, amigos ou da sociedade. A principal causa de estresse para o cuidador decorre das dificuldades resultantes do comportamento perturbador do paciente (Tognetti, 2004). Nesse contexto, o estresse se codifica como um termo que denota o estado gerado pela percepção de estímulos que provocam excitação emocional e que perturbam a homeostasia orgânica (Margis, Picon, Cosner \& Silveira, 2003).

O conceito de estresse envolve uma resposta biológica/hormonal ampla, porém, na literatura, o termo é utilizado como sinônimo de sobrecarga do cuidador. Outros termos utilizados são fadiga e impacto. Referem-se à presença de problemas, dificuldades ou eventos adversos que afetam significativamente a vida de pessoas que são responsáveis pelo paciente (Cassis et al., 2007, p. 497).

Tem-se demonstrado que cuidadores têm níveis mais elevados de depressão, hábitos inadequados de saúde, problemas fisiológicos, morbidade psiquiátrica ou médica, e mortalidade quando comparados aos não cuidadores (Cheng, Lau, Mak, Ng, Lam, Fung, Lai, Kwok \& Lee, 2012). As principais doenças físicas e psíquicas relatadas são: ansiedade, depressão, doenças cardiovasculares, além de declínio da função imunológica. Sendo assim, é perceptível a busca de meios de intervenção que possibilitem efeitos positivos tanto para o cuidador, como para o portador da Doença de Alzheimer. A presente revisão bibliográfica visa caracterizar quem normalmente arca com esses cuidados, os principais prejuízos do ponto de vista físico-patológico e suas respectivas intervenções como forma de melhora da qualidade de vida dos cuidadores, tendo como base o conteúdo das publicações científicas selecionadas.

\section{Método}


Foi realizada uma revisão bibliográfica das produções acerca do estresse em cuidadores de pacientes com Alzheimer nas bases eletrônicas: Scientific Electronic Library Online (ScieLO), PubMed, e Bireme (Biblioteca Virtual em Saúde). As palavras chaves / descritores utilizadas para a busca foram: estresse em cuidadores de Alzheimer, inseridos nos idiomas português e inglês. Os artigos foram selecionados de acordo com os seguintes critérios de inclusão: artigos que estivessem completos e disponíveis para leitura; nos idiomas português e inglês; publicados no período de 2006 a abril de 2013, sendo os mais recentes em termos de publicação na área. Os cuidadores a quem a revisão se refere podem ser familiares ou não. Os critérios de exclusão foram artigos fora do período proposto nos últimos anos, artigos não disponíveis integralmente para leitura ou que não tratassem da temática do estresse em cuidadores de pacientes com Alzheimer acerca de questões práticas da atividade, aspectos físico-patológicos e alternativas de intervenções.

A análise seguiu os preceitos da análise temática em que as informações foram agrupadas em três temas, analisadas e interpretadas. O primeiro tema se configura em caracterizar quem pratica essa atividade; o segundo em aspectos físico-psicopatológicos no contexto do cuidar; e o terceiro tema versa sobre os estudos de intervenção como alternativa de melhora dos sintomas de estresse e depressão.

\section{Resultados}

A partir destes critérios de busca foram encontradas no total 95 publicações (34 Bireme, 58 PubMed, 3 Scielo). Sendo que deste número, somente 32 se enquadraram aos critérios de inclusão e exclusão estabelecidos. Destes, dois foram publicados no ano de 2006, dois em 2007, sete em 2008, oito em 2009, sete em 2010, dois em 2011, três em 2012 e um de 2013. Sendo assim, o ano de 2009 foi o que houve mais publicações de acordo com a proposta desta revisão. Com relação às bases de dados, observou-se que a PubMed foi a que teve mais artigos mencionados (26), seguido da Scielo e Bireme, ambos com três artigos. Deve-se considerar que alguns artigos foram exibidos em mais de uma base de dados. Com relação à nacionalidade dos estudos, vinte e dois foram realizados nos Estados Unidos; sete no Brasil, um na Itália, República Checa e Holanda.

Dos 32 artigos, nove tratavam da prática do cuidar no aspecto de quem pratica essa atividade. Ou seja, discutiam sobre investigação, compreensão, e análise dos aspectos significativos na prestação de cuidados aos pacientes com DA. Destes nove, oito trazem essa prática do cuidar na visão dos familiares, e apenas um não define 0 tipo de cuidador em questão. Geralmente o cuidador é o membro da 
família que provê os cuidados ao paciente, outrora definido como o indivíduo que residia com o paciente e fosse atuante por mais de seis meses, ou ainda, como o principal responsável por prover ou coordenar os recursos requeridos pelo paciente (Cassis et al., 2007). Nesse sentido, os artigos retratam basicamente que essa prática é inicialmente responsabilidade da família, que por sua vez são quem detecta os primeiros sinais da morbidade (Almeida, Leite \& Hildebrandt, 2009; Wegierek, 2012). Com avanços na gestão de doenças crônicas, o papel da família dos pacientes com Alzheimer está se tornando mais complexo e exigente (Vitaliano, Zhang, Young, Caswell, Scanlan \& Echeverria, 2009), sendo uma questão que envolve todos os seus membros que passam a adquirir relevância no acompanhamento e apoio desse doente (Valim, 2010).

Outros 13 artigos se referem aos aspectos físico-psicopatológicos referentes a esta atividade. Todos tratam do estresse, como sintoma, ou como desencadeador de outros problemas físico-psicopatológicos, especialmente nos cuidadores familiares, que convivem com a sobrecarga física e psíquica que, não raro, levam a uma má qualidade de vida (Vilela \& Caramelli, 2006), evidenciando que o declínio do paciente e suas demandas específicas são fatores importantes de estresse para o cuidador. Estes apresentam altos índices de sintomas psiquiátricos - especialmente a depressão, prejuízos no sistema imunológico, assim como conflitos familiares (Cruz \& Hamdan, 2008). Além disso, os cuidadores de idosos portadores de demência apresentam um pior julgamento sobre a própria saúde, sentem-se mais estressados e com a vida afetiva e social mais limitada do que cuidadores de idosos não portadores de demência (Grafstrom, Fratiglioni, Sandman \& Winblad, 1992 apud Cruz \& Hamdan, 2008). Também há relação proporcional quanto ao estágio da doença do paciente e os sintomas de distúrbios de sono e fadiga crônica do acompanhante (Zverová, 2012).

No que diz respeito às temáticas de propostas de intervenção estão em nove artigos desta revisão. A maioria das intervenções realizadas são em sua maioria demonstradas com base em um grupo controle e um grupo experimental; que tanto intervém nos próprios pacientes como nos cuidadores. Dos nove artigos, oito versam sobre quando a intervenção é direcionada aos cuidadores demonstrando um efeito positivo na diminuição dos sintomas de estresse. Estes basicamente se sustentam em promover ao cuidador conhecimentos sobre o mal de Alzheimer, psicoeducação, aconselhamento psicológico ou propriamente um processo de psicoterapia com posterior avaliação da redução de danos (Glueckauf \& Davis, 2012; Cheng et al.,2012; Blom, Bosmans, Cuijpers, Zarit \& Pot, 2013), a abordagem mais citada é a Terapia Cognitiva Comportamental para controle do estresse e sintomas depressivos (Cheng et. al., 2012; Glueckauf et al., 2012; Blom et al., 2013). Outra forma de intervenção é a prática 
de exercícios físicos com os acompanhantes, as caminhadas obtiveram relação positiva com a melhora da qualidade de vida (McCurry, Pike, LogsdonVitielo, Larson \& Teri, 2010). E também o uso de medicamento como atenuador dos sintomas, como no caso do Escitalopram que demonstrou minimização na sintomatologia depressiva, resistência ao estresse e melhora da qualidade de vida. As formas que essas intervenções chegam aos cuidadores são diversificadas, tanto são consideradas válidas as clássicas intervenções presenciais, como por telefone e internet.

E os outros dois artigos discutem sobre quando a intervenção ocorre tanto no cuidador quanto no paciente, trazendo benefícios para ambos os lados. Dentre as opções, a intervenção no sistema motor dos pacientes, com exercícios aeróbicos (flexibilidade, força e agilidade) e exercícios funcionais de equilíbrio, demonstra significativa melhora em sua condição neuropsiquiátrica, tendo como consequência a redução dos sintomas de exaustão dos cuidadores. E a hospitalização do paciente com relação positiva com a melhora do estado de saúde do cuidador (Gaugler, Mittelman, Hepburn \& Newcomer, 2009). Outra intervenção com origem no paciente é sua hospitalização, como forma de prever se há melhora no estado de saúde do cuidador, demonstrando aproximação entre essas duas variáveis (Gaugler et al., 2009).

\section{Discussão}

Estudos sobre cuidadores de pacientes psiquiátricos surgiram pela primeira vez na década de 1960, seguido por estudos sobre cuidadores de idosos frágeis, e na década de 1980 e 1990 pela pesquisa sobre cuidadores de idosos com demência, incluindo pacientes com Doença de Alzheimer. Por isso, têm-se revelado que cerca de $40 \%$ dos indivíduos com 65 anos ou mais precisam de algum tipo de assistência com pelo menos uma tarefa, tais como compras, finanças, preparar refeições ou fazer a limpeza (Cachioni, Lima-Silva, Ordonez, GaloTiago, Alves, Suzuki \& Falcão, 2011). Em menor proporção (10\%) necessitam de ajuda para realizar tarefas básicas, como tomar um banho, vestir-se, usar o banheiro, alimentar-se ou entrar e sair de cadeiras e camas (Karsch, 2003); esses dados refletem as preocupações de mais seis milhões de indivíduos e famílias (IBGE, 2002).

\subsection{Quem pratica esta atividade}

Tendo como foco a prática do cuidado e os nove textos que comporam essa temática da revisão, é possível verificar que fatores de gênero, geracional e de parentesco influenciam na atribuição da 
tarefa de cuidar; além de outros aspectos como viver sob o mesmo teto, com mesmos meios financeiros e tempo disponível, os laços afetivos, aspectos da personalidade do cuidador, relacionamento passado com a pessoa idosa, a razão e a capacidade de se doar, também afetam essa disponibilidade (Cachioni et al., 2011). A tarefa de cuidar de indivíduos com DA normalmente recai sobre os membros da família, que podem ser seus responsáveis por muitos anos (Lindsey \& Buckwalter, 2009), tornando os idosos dependentes de alguma forma.

Esta postura entre idosos e familiares muitas vezes se resume a uma questão de cultura, uma vez que a maioria das sociedades considera a interação entre as gerações como o esteio da sua (Fonseca \& Soares, 2008). Ainda assim, a natureza de longo prazo de muitas doenças relacionadas à demência levou à caracterização da família cuidadora como uma 'carreira', com transições que ocorrem como progresso da doença, necessidades e capacidades de mudança, e alterações quanto ao ambiente de cuidados (Aneshensel, Pearlin, Mullan, Zarit \& Whitlatch, 1995 apud Nikzad-Terhune, 2010). A transição de um ambiente de enfermagem para 0 ambiente doméstico, por exemplo, é um processo complexo para cuidadores familiares de adultos mais velhos. Embora a colocação de home care possa aliviar certos cuidados e responsabilidades, pode também desencadear novos estressores (Nikzad-Terhune, Anderson, Newcomer \& Gaugler, 2010). Apesar disso, para alguns familiares o carinho e a vontade de ficar junto da pessoa que agora necessita de atenção, os impulsiona, motivando-os para realizar o cuidado. Sabese que o processo de cuidar engloba a compreensão de si e do outro, desse modo, o cuidado é tido como um encontro, em que a fragilidade do momento deve ser suportada, revelando-se como algo desafiador e que deve ser vivenciado diariamente (Almeida, Leite \& Hildebrandt, 2009).

Tendo isso como base, pesquisas têm evidenciado que, em sua maioria, os cuidadores são do sexo feminino, especialmente esposas e filhas do paciente, evidenciando que a mulheres exercem o papel de cuidar como uma função de raízes históricas, sociais, culturais e afetivas (Karsch, 2003; Colomé, Marqui, Janh, Resta, Carli, Winck \& Nora, 2011). Como ainda são frequentes na sociedade as mulheres que, além de muitas vezes ocupar outros postos de trabalho, ainda têm tempo para se dedicar a casa, aos filhos e ao esposo, acredita-se que o sexo feminino possa desempenhar essa tarefa com maior facilidade (Colomé et al., 2011), fazendo com que elas chegam a representar até $94 \%$ dos cuidadores (Reis \& Ceolim, 2007). Por outro lado esta atividade, associada ao trabalho feminino, problematiza a ideia do cuidar como categoria que pode caracterizar negativamente a condição de vida das mulheres, colocando em evidência a relevância do trabalho "doméstico" como obrigação puramente 
feminina, no sentido de reivindicar igualdade e partilha dessas tarefas entre os sexos (Carvalho, 2007).

Também há evidências de artigos voltados para a prática do cuidado numa perspectiva étnica. Um deles pretende comparar os cuidadores negros e brancos de familiares com Doença de Alzheimer em duas medidas gerais: emoções positivas e emoções negativas, sugerindo que os cuidadores negros relatam menos sintomas depressivos ao longo do tempo do que os brancos (Skarupski, Mccann, Bienias \& Evans, 2009). Da mesma forma há tendência de que a raça ou etnia possa moderar o impacto dos recursos sobre a tensão intrapsíquica, entretanto foi na raça caucasiana em que houve relatatos de aspectos intrapsíquicos mais negativos do que o africano ou hispânicos que demonstram contar com o suporte social como alternativa para controle do estresse (Hilgeman, Durkin \& Sun, 2008).

\subsection{Aspectos físicos- psicopatológicos no contexto do cuidar}

Os artigos que tratam dos aspectos físicos- psicopatológicos têm como foco os sintomas de estresse, uma vez que em sua maioria este se associa a outros problemas de saúde, como: ansiedade, depressão, cardiopatias e problemas imunológicos. Estudos com grupos-controle evidenciam algumas diferenças entre cuidadores de idosos demenciados e outros grupos populacionais, em estudo comparativo entre cuidadores de três grupos geriátricos diferentes pacientes com DA, pacientes com demência vascular e idosos não demenciados - revelou que o impacto sofrido pelos cuidadores é maior nos de portadores de DA (Rainer, Jungwisth \& Kruger-Rainer, 2002 apud Cruz \& Hamdan, 2008). Nesse sentido, cuidadores de Alzheimer são os que possuem indicação de experiências a níveis significativamente mais elevados de sintomas psiquiátricos em relação a outras morbidades que requerem esse acompanhamento (Ory, 1999 apud Mausbach, Patterson \& Grant, 2008a). Isto não é surpreendente considerando 0 alto nível de responsabilidade experimentado pelos cuidadores (Mausbach, Patterson \& Grant, 2008).

Outros estudos indicam que os cuidadores de pacientes com demência apresentam maiores índices que cuidadores de pacientes com depressão, AVC ou idosos hígidos (Garrido \& Almeida, 1999). Contam, então com uma maior predisposição para sintomas psiquiátricos, problemas de saúde, conflitos familiares e problemas no trabalho, se comparados a pessoas da mesma idade que não exercem essa função (Cerqueira \& Oliveira, 2002). Ainda assim, há evidencia de que cuidadores de idosos portadores de DA apresentam um pior julgamento sobre a própria saúde, sentem-se mais estressados e com a vida afetiva e social mais limitada que acompanhantes de outros idosos (Grafstromet, Fratiglioni, Sandman 
\& Winblad, 1992, apud Cruz \& Hamdan, 2008). A resposta fisiológica ao estresse em geral inclui a secreção de hormônios neuroendócrinos e catecolaminas pelo eixo hipotálamo-hipófise-adrenal no sistema nervoso simpático, que por sua vez modula funções de vários tipos de células, incluindo células do sistema imunológico (Pederson, 2001 apud Damjanovic, 2007). Nesse sentido, o estresse psicológico crônico tem um impacto negativo sobre a função das células imunológicas e pode acelerar o envelhecimento.

Somado a isso, a elevada ativação simpática é evidenciada pelo aumento das catecolaminas circulantes. É bem estabelecido que as situações de forma aguda de estresse ativam os nervos simpáticos, resultando em um aumento de circulação de plasma e norepinefrina (NE) (Mills, Adler \& Dimsdale, 2004; Black \& Garbutt, 2002, apud Roepke, Mausbach, Aschbacher, Ziegler, Dimsdale, Mills, Von Känel, Ancoli-Israel, Patterson \& Grant, 2008). Por sua vez, catecolaminas podem produzir mudanças fisiológicas, tais como aumento do metabolismo, frequência cardíaca e pressão arterial (Roepke et al., 2008), o que justifica o risco de desenvolver hipertensão ou doenças cardiovasculares entre os cuidadores (Mausbacha, Patterson \& Grant, 2008). Pessoas que avaliam a si mesmos como incapazes de gerir estressores, ou seja com baixo domínio em situações adversas, podem ter maior excitação fisiológica impulsionado por excitação simpática. Em contraste, àqueles que se avaliam como capaz de gerir eventos estressores podem tanto perceber o evento como menos ameaçador e ter uma experiência de controle, resultando em menos excitação fisiológica (Roepke et al., 2008).

Por esses e outros motivos, cuidadores de DA que têm estresse crônico demonstram comprometimento endotelial, e têm sido associados com risco aumentado de eventos cardiovasculares. Estes resultados sugerem um mecanismo potencial pelo qual a tensão crônica da prestação de cuidados leva a resultados negativos na saúde (Mausbach et al., 2010). E, assim sendo, o maior número de problemas de comportamento e reações negativas dos cuidadores são associados com maior índice pró-coagulante que podem ser os principais fatores de risco cardiovascular (Von Känel, Mausbach, Dimsdale, Mills, Patterson, Ancoli-Israel, Ziegler, Roepke, Allison \& Gran , 2010a).

Nesse sentido, outro indicativo importante de ser mencionado se refere ao fato de que cuidadores geralmente dormem pouco, sendo uma característica própria da atividade pela necessidade de atenção como forma de evitar acidentes caso o paciente necessite de algum recurso durante a noite, o que pode ocasionar o aumento da atividade de coagulação e disfunção endotelial e contribuindo para uma maior tendência para doenças cardiovasculares (Von Känel et al. 2010b), incluindo acidente vascular cerebral, (Elwood, Hack, Pickering, Hughes \& Gallacher, 2006; Phillips \& Mannino, 2007 apud 
Mills, 2009) que se dá pelas elevações nos níveis de circulação de hipercoagulabilidade e biomarcadores inflamatórios da doença cardiovascular (Mills et al., 2009).

Um aspecto psicopatológico consonante aos outros aspectos já explicitados é a depressão, já que os níveis de sintomas depressivos entre os cuidadores são altos, podendo chegar a até $55 \%$ de prevalência nos que desempenham essa função (Garrido \& Almeida, 2004). Os resultados atuais sugerem que os sintomas depressivos persistentes, mesmo quando eles estão em termos de gravidade subclínica, estão fortemente associados com ativação plaquetária em resposta ao estresse agudo (Schulz, Mcginnis, Zhang, Martire, Hebert, Beach, S. Zdaniuk, Czaja \& Belle, 2008). O exagerado número de plaquetas em respostas ao estresse agudo também pode se constituir em um importante mecanismo para as associações potenciais entre afeto negativo e eventos cardiovasculares ou mortalidade (Aschbacher, Roepke, Von Känel, Mills, Mausbach, Patterson, Dimsdale, Ziegler, Ancoli-Israel \& Grant, 2009). Além disso, outros conteúdos também aparecem prejudicados nessa função e se agravam de acordo com a evolução da DA, sugerindo correlação positiva entre 0 aspecto neurológico do paciente e 0 comprometimento da qualidade de vida. Melhor rendimento no Mini Exame de Estado Mental (MEEM), instrumento tipicamente utilizado para avaliações em portadores de DA, é relacionado a melhores indicativos de sintomas de depressão nos acompanhantes no Inventário Beck de Depressão (IBD) e nos escores de vitalidade e sintomas físicos e emocionais do da escala de qualidade de vida SF36 (Pinto, Barbosa, Ferreti, Souza, Fram \& Belasco, 2009). Além da capacidade do paciente se relacionar aos indicativos de depressão e qualidade de vida do cuidador, há também aumento dos sintomas de distúrbios de sono e fadiga crônica com o aumento das dificuldades experienciadas pelo paciente (Zverová, 2012).

\subsection{Estudos de intervenção}

$\mathrm{Na}$ busca por interromper ou inibir os problemas já citados, é perceptível o aparecimento de artigos que discutem a importância de se buscar barreiras artificiais ou não com intuito de minimizar essa problemática. Por isso, dos estudos que propõem intervenções, quatro artigos tratam de intervenção diretamente nos cuidadores. Dois desses se referem a uma intervenção que diz respeito ao uso de chamadas telefônicas como sessões terapêuticas, com um período mínimo de seis meses para obtenção de resultados de intervenção. Outro deles se remetem à intervenção nos cuidadores etnicamente diversificados e o outro a uma intervenção que avaliou os custos e benefícios, como base para produzir uma unidade adicional de benefício por dia. 
Observou-se que a intervenção multicomponente conta com técnicas como aconselhamento e suporte telefônico, estruturadas e adaptadas aos perfis individuais, podendo aumentar a qualidade de vida dos cuidadores de demência. Considerando a diversidade etnica, cuidadores hispânicos e caucasianos, participantes das intervenções, experimentaram melhora significativa na qualidade de vida do que aqueles do grupo controle; sugerindo que a depressão clínica foi menor entre os cuidadores do grupo de intervenção (Belle, Burgio, Burns, Coon, Czaja, Gallagher-Thompson, Gitlin, Klinger, Koepke, Lee, Martindale-Adams, Nichols, Schulz, Stahl, Stevens, Winter \& Zhang, 2006) Os custos emocionais e físicos da prestação de cuidados nos casos de demência são relevantes e a intervenção se mostra capaz de amenizar esses custos emocionais e físicos (Nichols, Chang, Lummus, Burns, Martindale-Adams, Graney, Coon \& Czaja, 2008). Essas intervenções centram-se em fornecer ao cuidador informações sobre a enfermidade de seus pacientes, psicoeducação, aconselhamento psicológico ou mesmo acompanhamento psicoterápico breve (Glueckauf et al., 2012; Cheng et al.,2012; Blom et al., 2013), das opções de psicoterapia breve, a que se destaca na literatura como alternativa de controle de estresse também nesses casos é a Terapia Cognitivo Comportamental (TCC) (Cheng et. al., 2012; Glueckauf et al., 2012; Blom et al., 2013). Nesse ponto a TCC tem contribuído por ser uma modalidade de resultados a curto prazo que permite o trabalho desde os pensamento automáticos até as crenças centrais do indivíduo, sempre considerandos os eventos desencadeadores dos sintomas (Wright, Basco, \& Thase, 2008).

Além disso, um cuidador mais informado dispõe de mais estratégias que possam facilitar sua atividade de maneira a tornar aquele trabalho menos estressante, já que há um maior arcabouço de conhecimentos de como lidar com situações até mesmo específicas do quadro. As intervenções de aconselhamento psicológico ou psicoterapia breve são facilitadoras por auxiliar na elaboração os sintomas de estresse e viabilizar a aquisição de novas estratégias de enfrentamento mais adequadas e por consequência menos danosas para sua saúde (Roepke et al., 2008). Diferentemente dessa intervenção, outra proposta consiste em intervenção medicamentosa apartir da avaliação do potencial do antidepressivo escitalopram na melhora da depressão, resistência ao estresse e qualidade de vida em cuidadores familiares. $O$ uso do escitalopram resultou em melhora da depressão, de angústia, e qualidade de vida (Lavretsky, Siddarth \& Irwin, 2010).

O que parece ser diversificado nos estudos sobre intervenção são os meios pelos quais estas chegam aos seus usuários, sugerindo como possibilidade, além dos comuns encontros presenciais, os contatos telefônicos ou através da internet. Em comparações quanto à intervenção em sintomas de depressão e status de saúde de 
cuidadores atendidos presencialmente e por telefone, foram observados indicativos de melhoras mais significativas naqueles atendidos por telefone, levantando a hipótese de mais estudos para verificação de variáveis que podem ter influenciado esse resultado (Glueckalf et al.,2012). Já a intervenção pela internet consistia em oito sessões e uma última para consolidação, realizadas por um psicólogo, com o foco na prevenção e diminuição da sintomatologia depressiva e do estresse, demonstrando resultados satisfatórios quanto aos seus objetivos. Essas novas modalidades de aproximação do cuidador permitem que se possa ter um acompanhamento de qualidade sem necessariamente ter uma reserva maior tempo para ir aos consultórios ou centros de saúde, mostrando-se como alternativas de baixo custo financeiro, tempo e boa eficácia. Otimizando a adesão do cuidador aos programas de intervenção pelas facilidades propostas, sem contar que em muitos casos há certa dificuldade em se ausentar dos cuidados com o paciente.

Outras formas de intervenção sugerem ações nos pacientes com reflexos no cuidador, como no caso de atividades motoras com a prática de exercícios funcionais de equilibrio e exercícios aeróbicos que estimulam a flexibilidade, força e agilidade dos pacientes que ocasioraram uma significativa melhora de suas condições neuropsiquiátricas, e consequentemente, a redução dos sintomas de exaustão dos cuidadores (Stella, Canonici, Sebastião, SantosGalduroz, Cação \& Gobbi,2011). Outra opção é a institucionalização do paciente com o objetivo de verificar as mudanças na sobrecarga no cuidador e nos sintomas depressivos como forma de diferenciar se os referidos sintomas estão realmente relacinados à pratica laboral ou se persistem, podendo indicar outras origens. Nesse experemento foram observados menos relatos de sintomas de estresse e depressão após a internação dos pacientes, sugerindo suas causas ou agravamento na prática do cuidar (Gaugler et al., 2009). Mesmo que essas práticas tenham se mostrado eficázes, há limitações quanto a sua durabilidade. Por ser uma doença progressiva, não tem como prever por quanto tempo após a prática dos exercícios o paciente voltará a ter as mesmas dificuldades de antes, ou até sua piora. Da mesma forma, a menos que o paciente esteja em estado crítico e necessite de cuidados médicos constantes, não permanecerá internado durante todo o curso da doença, retornando ao lar quando possível e assim retomando toda a rotina anterior à sua internação, considerando que a intervenção se restrinja somente a essa prática. Pode-se sugerir que os objetivos das intervenções foram todos cumpridos e comprovaram as hipóteses iniciais. Mas, vale ressaltar que nenhum método de estudo seria suficiente para que se esgotassem os modos de se pensar a respeito de tal assunto. 


\section{Considerações finais}

Sugere-se que um dos aspectos mais importantes desta revisão foi observar que o estresse nos cuidadores é um ponto que se expande em muitos outros problemas que desencadeiam comprometimentos na qualidade de vida, do ponto de vista físico, mental, psicológico e emocional. Por isso, se faz preponderante uma rede de suporte profissional que possa ao menos minimizar parte dessa problemática por meio de intervenções mais eficazes e continuadas. Como podemos identificar, essa revisão carece de uma completude, uma vez que suas principais limitações são: número reduzido de base de dados utilizadas, o que poderia fornecer mais estudos com seus diferentes referenciais. Outra limitação foi o fato de não termos deixado claro em todas as temáticas de que tipo de cuidador estava em questão - familiar ou não - talvez por esta também ter sido uma limitação dos artigos revisados.

No entanto essa revisão contribui para a elucidação dos principais comprometimentos emocionais, e até mesmo físicos, dos quais o cuidador do paciente portador de Alzheimer está exposto. Auxiliando na prevenção ou agravamento desses sintomas na medida em que discute diferenciados tipos de intervenções a curto e longo prazo como alternativa de não só favorecer o estado de saúde do cuidador, como também proporcionar garantias de maior segurança dos pacientes. Um cuidador equiliibrado mentalmente e físicamente poderá oferecer um serviço de melhor qualidade.

\section{Referências}

Almeida, K. S., Leite, M. T. \& Hildebrandt, L. M. (2009). Cuidadores familiares de pessoas portadoras de doença de Alzheimer: revisão da literatura. Revista Eletrônica de Enfermagem [Internet], 11(2), 403412.

Aschbacher, K., Roepke, S. K., Von Känel, R., Mills, P. J., Mausbach, B. T., Patterson, T. L., Dimsdale, J. E., Ziegler, M. G. , Ancoli-Israel, S. \& Grant, I. (2009). Persistent Versus Transient Depressive Symptoms in Relation to Platelet Hyperactivation: A Longitudinal Analysis of Dementia. Journal of Affective Disorders, 116(1-2), 80-87. Belle, S. H., Burgio, L., Burns, R., Coon, D.; Czaja, S. J., GallagherThompson, D., Gitlin, L. N., Klinger, J., Koepke, K. M., Lee, C. C., Martindale-Adams, J., Nichols, L., Schulz, R., Stahl, S., Stevens, A., Winter, L. \& Zhang, S. (2006). Enhancing the Quality of Life of Dementia Caregivers from Different Ethnic or Racial Groups. Annales medicinae internae Fenniae, 145(10), 727-738.

Blom, M. M., Bosmans, J. E., Cuijpers, P., Zarit, S. H. \& Pot, A. M. (2003). Effectiveness and cost-effectiveness of an internet 
intervention for family caregivers of people with dementia: design of a randomized controlled trial. BMC Psychiatry, 13(17), 1-7.

Cachioni, M., Lima-Silva, T. B., Ordonez, T. N., Galo-Tiago, J., Alves, A. R., Suzuki, M. Y. \& Falcão, D. V. S. (2011). Elderly patients with Alzheimer's disease and their family relationships: caregiver perspectives. Dementia \& Neuropsychologia, 5, 114-122.

Carvalho, M. I. L. B. (2007). Entre cuidados e cuidadores: o feminino na configuração da política de cuidados às pessoas idosas. Revista Campus Social, 3 (2-3), 269-280.

Cassis, S. V. A., Karnakis, T., Morais, T. A., Curiati, J. A. E., Quadrante, A. C. R. \& Magaldi, R. M. (2007). Correlação entre o estresse do cuidador e as características clínicas do paciente portador de demência. Revista da Associação Médica Brasileira, 53(6), 497501.

Cerqueira, A. T. A. R. \& Oliveira, N. I. L. (2002). Programa de apoio a cuidadores: uma ação terapêutica e preventiva na atenção à saúde dos idosos. Psicologia USP, 13 (1), 133-150.

Colomé, I. C. S., Marqui, A. B. T., Janh, A. C., Resta, D. G., Carli, R., Winck, M. T. \& Nora, T. T. D. (2011). Cuidar de idosos institucionalizados: características e dificuldades dos cuidadores. Revista Eletrônica de Enfermagem, 13(2), 306-312.

Cheng, S. T., Lau, R. W. L., Mak, E. P. M., Ng, N. S. S., Lam, L. C. W., Fung, H.H., Lai, J. C. L.; Kwok, T. \& Lee, D. T.. F. (2012). A benefit-finding intervention for family caregivers of persons with Alzheimer disease: study protocol of a randomized controlled trial. Trials, 13(98), 1-10.

Cruz, N. \& Hamdan, A. C. (2008). O impacto da doença de Alzheimer no cuidador. Psicologia em Estudo, 13 (2), 223-229.

Damjanovic, A. K., Yang, Y., Glaser, R., Kiecolt-Glaser, J. K., Nguyen, H., Laskowski, B. \& Zou, Y. (2007). Beversdorf Dq, Weng N. Accelerated Telomere Erosion Is Associated with a Declining Immune Function of Caregivers of Alzheimer's Disease Patients. Journal of immunology, 179 (6), 4249-4254.

Ferrara, M., Langiano, E., Brango, T.D., Vito, E. D., Cioccio, L. D.\& Bauco, C. (2008). Prevalence of stress, anxiety and depression in with Alzheimer caregivers. Health and Quality of Life Outcomes, 6 (93), 1-5.

Fonseca, A. M. \& Soares, E. (2008). O processo saúde-doença e o cuidado domiciliário ao portador de doença de Alzheimer. Família, Saúde e Desenvolvimento, 8(2), 163-167.

Garrido, R. \& Almeida, O. P. (1999). Distúrbios de comportamento em pacientes com demência: impacto na vida do cuidador. Arquivos de Neuropsiquiatria, 57 (2), 427-434.

Gaugler, J. E. Mittelman, M. S., Hepburn, K. \& Newcomer, R. (2009). Predictors of Short and Long-Term Adherence to a Daily Walking 
Program in Persons with Alzheimer's Disease. Psychology Aging, 24(2), 385-396.

Glueckauf, R. L. \& Davis, W. S. (2012). Telephone-Based, CognitiveBehavioral Therapy for African American Dementia Caregivers With Depression: Initial Findings. Rehabilitation Psychology, 57(2), 124139.

Hilgeman, M. M., Durkin, D. W., Sun, F.; Decoster, J., Allen, R. S., Gallagher-Thompson, D. \& Burgio, L. D. (2008). Testing a Theoretical Model of the Stress Process in Alzheimer's Caregivers With Race as a Moderator. The Gerontologist, 49(2), 248-261.

Instituto Brasileiro de Geografia e Estatística [IBGE] (2002). Pesquisa Nacional por Amostra de Domicílios (PNAD): síntese de Indicadores. Recuperado em 25 setembro, 2011, de <http://www.ibge.gov.br/home/estatistica/populacao/trabalhoerendi mento/pnad2012/default_sintese.shtm>

Instituto Brasileiro De Geografia E Estatística IBGE. (2000) Pesquisa Nacional por Amostra de Domicílios - PNAD. Recuperado em 25 de setembro, 2011 de: <http://www.ibge.gov.br/home/estatistica/populacao/default_censo_ 2000.shtm>

Karsch, U. M. (2003). Idosos dependentes: famílias e cuidadores. Caderno de Saúde Pública, 19 (3), 861-866.

Lavretsky, H., Siddarth, P. \& Irwin, M. R. (2010). Improving depression and enhancing resilience in family dementia caregivers: a pilot randomized placebo-controlled trial of escitalopram. American J ournal of Geriatric Psychiatry, 18(2), 154.

Leitão, G. C. M. \& Almeida, D. T. (2000). O cuidador e sua qualidade de vida. Acta Paulista de Enfermagem, 13(1), 80-85.

Lindeboom J. \& Weinstein, H. (2004). Neuropsychology of cognitive ageing, minimal cognitive impairment, Alzheimer's disease, and vascular cognitive impairment. European Journal Pharmacology, 490(1-3), 83-86.

Lindsey, P. L. \& Buckwalter, K. C. (2009). Psychotic Events in Alzheimer's Disease. Journal of Gerontolological Nursing, 35(8), 2029.

Machado, J. C. B. Doença de Alzheimer. (2006). In Freitas EV, Py L, Cançado FAX, Doll J, Gorzoni ML. Tratado de geriatria e gerontologia. (2 ed, pp. 260-279). Rio de Janeiro: Guanabara Koogan.

Margis, R., Picon, P., Cosner, A. F. \& Silveira, R. O. (2003). Relação entre estressores, estresse e ansiedade. Revista de Psiquiatria do Rio Grande do Sul, 25, 65-74.

Mausbach, B. T., Aschbachera, K., Mills, P. J., Roepke, S. K., Von Känel, M. R., Patterson, T. L., Dimsdale, J. E., Ziegler, M. G., AncoliIsrael, S. \& Grant, I. (2008b). A 5-year longitudinal study of the relationships between stress, coping, and immune cell $\beta 2$-adrenergic receptor sensitivity. Psychiatry Research, 160(3), 247-255. 
Mausbach, B.T., Patterson, T.L. \& Grant, I. (2008a). Is Depression in Alzheimer's Caregivers Really Due to Activity Restriction? A Preliminary Mediational Test of the Activity Restriction Model. Journal of Behavior Therapy and Experimental Psychiatry, 39 (4), 459-466.

Mausbach, B. T., Roepke, S. K., Ziegler, M. G., Milic, M., Von Känel, R.; Dimsdale, J. E., Mills, P. J., Patterson, T. L., Allison, M. A., AncoliIsrael, S. \& Grant, I. (2010). Association Between Chronic Caregiving Stress and Impaired Endothelial Function in the Elderly. Journal of the American College of Cardiology, 55(23), 2599-2606.

Mccurry, S. M., Pike, K. C., Logsdon, R. G., Vitielo, M. V., Larson, E. B. \& Teri, L. (2010). Predictors of Short and Long-Term Adherence to a Daily WalkingProgram in Persons with Alzheimer's Disease. American Journal oof Alzheimers Disease and Other Dementias, 25(6), 505-512.

Mills, P. J., Ancoli-Israel, S., Von Känel, R., Mausbach, B. T., Aschbacher, K., Patterson, T. L., Ziegler, M. G., Dimsdale, J. \& Grant, I. (2009). Effects of Gender and Dementia Severity on Alzheimer's Disease Caregivers' Sleep and Biomarkers of Coagulation and Inflammation. Brain Behavior Immunology, 23(5), 605-610.

Nascimento, L. C., Moraes, E. R., Silva, J. C., Veloso, L. C. \& Vale, A. R. M. C. (2008). Cuidador de idosos: conhecimento disponível na base de dados LILACS. Revista Brasileira de Enfermagem, 61(4), 514-517.

Nichols, L.O., Chang, C., Lummus, A., Burns, R., Martindale-Adams, J., Graney, M.J., Coon, D.W. \& Czaja, S. (2008). The CostEffectiveness of a Behavior Intervention with Caregivers of Patients with Alzheimer's Disease. Journal of the American Geriatrics Society, 56(3), 413-420.

Nikzad-Terhune, K.A., Anderson, K.A., Newcomer, R. \& Gaugler, J. E. (2010). Do Trajectories of At-Home Dementia Caregiving Account for Burden After Nursing Home Placement? A Growth Curve Analysis. Social Work in Health Care, 49(8), 734-752.

Pinto, M. F., Barbosa, D. A., Ferreti, C. E. L., Souza, L. F., Fram, D. S. \& Belasco, A. G. S. (2009). Qualidade de vida de cuidadores de idosos com doença de Alzheimer. Acta Paulista de Enfermagem, 22(5), 652-577.

Reis, P. O. \& Ceolim, M. F. (2007). O significado atribuído a 'ser idoso' por trabalhadores de instituições de longa permanência. Revista da Escola de Enfermagem da Universidade de São Paulo, 41(1), 57-64.

Roepke, S. K., Mausbach, B. T., Aschbacher, K., Ziegler, M. G., Dimsdale, J. E., Mills, P. J., Von Känel, R., Ancoli-Israel, S., Patterson, T.L. \& Grant, I. (2008). Personal Mastery is Associated with Reduced Sympathetic Arousal in Stressed Alzheimer Caregivers. American J ournal of Geriatric Psychiatry, 16(4), 310-317. 
Schulz, R., Mcginnis, K. A., Zhang, S., Martire, L. M., Hebert, R. S., Beach, S. R., Zdaniuk, B., Czaja, S. J. \& Belle, S. H. (2008). Dementia Patient Suffering and Caregiver Depression. Alzheimer Disease and Associated Disorders, 22(2), 170-176.

Skarupski, K. A., Mccann, J. J., Bienias, J. L. \& Evans, D. A. (2009). Race differences in emotional adaptation of family caregivers. Aging Ment Health, 13(5), 715-724.

Stella, F., Canonici, A. P., Sebastião, G., Santos-Galduroz, R. F., Cação, J. C. \& Gobbi, L. T. B. (2011). Attenuation of neuropsychiatric symptoms and caregiver burden in Alzheimer's disease by motor intervention: a controlled trial. Clinics, 66(8), 1353-1360.

Tognetti, A. (2004). Le problematiche del Caregiver. Journal of Gerentology, 52(2004), 505-510.

Valim, M. D., Damasceno, D. D., Abi-Acl, L. C., Garcia, F. \& Fava, S.M.C. L. (2010). A doença de Alzheimer na visão do cuidador: um estudo de caso. Revista Eletrônica de Enfermagem, 12(3), 528-534.

Vilela, L. P. \& Caramelli, P. (2006). A doença de Alzheimer na visão de familiares de pacientes. Revista da Associação Médica Brasileira, 52(3), 148-152.

Vitaliano, P. P., Zhang, J., Young, H. M., Caswell, L. W., Scanlan, J. M. \& Echeverria, D. (2009). Depressed Mood Mediates Decline in Cognitive Processing Speed in Caregivers. The Gerontologist, 49(1), 12-22.

Von Känel, R., Ancoli-Israel, S., Dimsdale, J. E., Mills, P. J., Mausbach, B. T., Ziegler, M. F., Patterson, T. L. \& Grant, I. (2010b). Sleep and Biomarkers of Atherosclerosis in Elderly Alzheimer Caregivers and Controls. Gerontology, 56(1), 41-50.

Von Känel, R., Mausbach, B. T., Dimsdale, J. E., Mills, P. J., Patterson, T. L., Ancoli-Israel, S., Ziegler, M. G., Roepke, S. K., Allison, M. \& Grant, I. (2010a).Problem Behavior of Dementia Patients Predicts Low-Grade Hypercoagulability in Spousal Caregivers. J ournals of Gerontologys, 65(9), 1004-1011.

Wright, J.H., Basco, M.R. \& Thase, M.E. (2008). Aprendendo a terapia cognitivo-comportamental (1 ed). Porto Alegre: Artmed.

Zvěřová, M. (2012). Frequency of some psychosomatic symptoms in informal caregivers of Alzheimer's disease individuals. Prague's experience. Activitas Nervosa Superior Rediviva, 54(2), 77-132.

\section{Endereço para correspondência}

\section{Kamilla Sthefany Andrade de Oliveira}

Endereço Institucional: Avenida Senador Salgado Filho, 3000, Lagoa Nova, CEP 59078-970, Natal - RN, Brasil

Endereço eletrônico: millasthefany@hotmail.com

Marianna Carla Maia Dantas de Lucena

Endereço Institucional: Avenida Senador Salgado Filho, 3000, Lagoa Nova, CEP 59078-970, Natal - RN, Brasil

Endereço eletrônico: mariannacarla.lucena@gmail.com 
Kamilla Sthefany Andrade de Oliveira,

Marianna Carla Maia Dantas de Lucena, João Carlos Alchieri Estresse em cuidadores de pacientes com Alzheimer: uma revisão de literatura

\section{J oão Carlos Alchieri}

Endereço Institucional: Avenida Senador Salgado Filho, 3000, Lagoa Nova, CEP 59078-970, Natal - RN, Brasil

Endereço eletrônico: jcalchieri@gmail.com

Recebido em: 18/06/2012

Reformulado em: 08/05/2013

Aceito para publicação em: 13/05/2013

Acompanhamento do processo editorial: Deise Maria Leal Fernandes Mendes

\section{Notas}

* Aluna do curso de Psicologia, da Universidade Federal do Rio Grande do Norte / UFRN. Natal / RN, Brasil.

** Psicóloga. Mestre em Psicologia pela Universidade Federal do Rio Grande do Norte / UFRN. Natal / RN, Brasil.

*** Professor Doutor associado do departamento de Psicologia da Universidade Federal do Rio Grande do Norte / UFRN, e orientador dos programas de pósgraduação em Psicologia e Ciências da Saúde. Natal / RN, Brasil. 